\title{
Acute immobilisation facilitates premotor preparatory activity for the non-restrained hand when facing grasp affordances
}

\author{
Simone Kühn *, Anika Werner, Ulman Lindenberger, Julius Verrel \\ Max Planck Institute for Human Development, Center for Lifespan Psychology, Lentzeallee 94, 14195 Berlin, Germany
}

\section{A R T I C L E I N F O}

\section{Article history:}

Accepted 2 February 2014

Available online 12 February 2014

\section{Keywords:}

Short-term immobilisation

Plasticity

Cortical reorganisation

fMRI

Affordance

\begin{abstract}
A B S T R A C T
Use and non-use of body parts during goal-directed action are major forces driving reorganisation of neural processing. We investigated changes in functional brain activity resulting from acute short-term immobilisation of the dominant right hand. Informed by the concept of object affordances, we predicted that the presence or absence of a limb restraint would influence the perception of graspable objects in a laterally specific way. Twenty-three participants underwent fMRI scanning during a passive object-viewing task before the intervention as well as with and without wearing an orthosis. The right dorsal premotor cortex and the left cerebellum were more strongly activated when the handle of an object was oriented towards the left hand while the right hand was immobilised compared with a situation where the hand was not immobilised. The cluster in the premotor cortex showing an interaction between condition (with restraint, without restraint) and stimulus action side (right vs. left) overlapped with the general task vs. baseline contrast prior to the intervention, confirming its functional significance for the task.

These results show that acute immobilisation of the dominant right hand leads to rapid changes of the perceived affordance of objects. We conclude that changes in action requirements lead to almost instantaneous changes in functional activation patterns, which in turn may trigger structural cortical plasticity.
\end{abstract}

(C) 2014 Published by Elsevier Inc.

\section{Introduction}

Plasticity and reorganisation are intrinsic properties of the human brain across the entire lifespan. In the sensorimotor domain, this adaptability has been investigated using two main routes: the observation of changes related to increases of sensory input or motor output (PascualLeone et al., 2005) as well as the deprivation of similar input and output (Bassolino et al., 2012; Taub et al., 2006). Regarding the latter approach, a standard manipulation is the immobilisation of limbs for a limited period of time, either experimentally induced or as a consequence of fractures, which allows investigating the central effects of non-use. Studies focussing on long-term immobilisation over periods of several weeks observed structural decreases in contralateral primary motor areas (Granert et al., 2011; Langer et al., 2012) and increases in cortical thickness and white matter properties on the ipsilateral side (Langer et al., 2012). Functionally, decreases in corticomotor excitability (Granert et al., 2011) and increases in intracortical inhibition (Clark et al., 2010) were detected by means of transcranial magnetic stimulation (TMS) over the contralateral motor area after long-term immobilisation. Likewise, reductions of blood flow in motor areas of the non-used side

\footnotetext{
* Corresponding author at: Max Planck Institute for Human Development, Germany. E-mail address: kuehn@mpib-berlin.mpg.de (S. Kühn).
}

have been demonstrated using positron emission tomography (Coert et al., 2009).

Even short-term immobilisation in the range of days or only several hours can affect the central representation of movement. Behaviourally, changes in reaching to grasp (Bassolino et al., 2012), anticipatory postural adjustments of the elbow and the shoulder (Bolzoni et al., 2012), and in interjoint coordination were observed. The resulting changes were smaller in amplitude but similar in quality to those reported in deafferented patients (Moisello et al., 2008). Four days of immobilisation have been shown to decrease corticospinal excitability in the contralateral motor cortex (Avanzino et al., 2011; Facchini et al., 2002; Ngomo et al., 2012) as well as inhibitory influence onto the ipsilateral motor cortex (Avanzino et al., 2011) when probed by TMS. By means of functional magnetic resonance imaging (fMRI), short-term immobilisation effects were shown in the somatosensory cortex in association with discrimination thresholds (Lissek et al., 2009). Another group has shown an increased cortical activation during tactile stimulation of the non-immobilised hand, particularly in the ipsilateral somatosensory cortex, after only $72 \mathrm{~h}$ of immobilisation (Weibull et al., 2011).

The effects of long- and short-term immobilisation reported so far are based on non-use periods of at least several hours to months. In contrast, the present study aimed at investigating more rapid effects of immobilisation. Participants' dominant right hand was immobilised by means of an orthosis immediately preceding data acquisition. 
Hence, the focus of this study is on the influence of knowing that one cannot move the right hand, rather than extensive experience with the inability to move the hand. While the effects of immobilisation observed in previous studies were strongly tied to action execution or somatosensory stimulation, we aimed at investigating the acute effects of immobilisation in a more abstract cognitive task domain. During the task, objects with handles that were either oriented to the right or left side were shown, and participants were instructed to passively observe them. Based on the concept of affordances, it has been argued that humans perceive objects in terms of the ways in which they can be used in action (Gibson, 1977; Prinz, 1997). Multiple studies have shown evidence for the automatic extraction of affordances when seeing objects and the spontaneous activation of associated action representations (Castiello, 1999; Craighero et al., 1996; Gentilucci, 2002; Grèzes et al., 2003). Based on this notion, we hypothesized that acute immobilisation of the dominant right hand would facilitate preparatory motor activity for the left hand when the handle is oriented to the left side compared to an unrestrained condition.

\section{Methods}

\section{Participants}

Twenty-three healthy young adults (age: mean $=25.43$ years, ranging from 20 to 35,11 females) participated after having given a written informed consent. The study was conducted according to the Declaration of Helsinki, with approval of the German Psychological Society ethics committee. All participants had normal or corrected-tonormal vision, and no history of neurological, major medical, or psychiatric disorder (as assessed by means of the MINI, Lecrubier et al., 1997) nor did they use psychotropic drugs during the last year. Moreover they did not wear a hand cast or an orthosis during the last five years. All participants were right-handed as assessed by the Edinburgh handedness questionnaire ( mean score $=88.9 ; \mathrm{SD}=6.9$, Oldfield, 1971).

\section{Materials}

To immobilise the right hand of the participants we used a commercially available orthosis (Model 28P44, Ottobock, Duderstadt, Germany). The hand was fixed in a way that prevented extension and flexion of the fingers, the thumb and the wrist. A piece of cloth was placed between the skin and the orthosis. To minimize differences in tactile stimulation between conditions, the same piece of cloth was also placed under the hand when the unrestrained measurement without the orthosis was performed.

For the object task, we selected 20 pictures of objects such as a cup, a hair dryer, a teapot, or scissors. Each object was presented in the original and a mirror-reversed version, suggesting either a right-hand or lefthand grasp (handle facing right or left side). This resulted in a total of 40 visual stimuli.

\section{Object task}

During the experiment, participants were shown the object pictures. They were instructed to watch the pictures carefully. Each trial started with a presentation of one of the pictures for $3 \mathrm{~s}$. After a jitter interval between 5 and $7 \mathrm{~s}$ (varied in steps of $500 \mathrm{~ms}$ ) the next object was presented (Fig. 1A). The experiment consisted of 3 runs, each containing 40 trials, with an overall duration of approximately 6 min per run.

Before the use of the orthosis was introduced to the participants, the object task was performed in the scanner once to localize the regions involved in task performance (this phase is called localizer phase from now on). Subsequently, participants completed the object task once with and once without the orthosis. The order of these latter two conditions was counterbalanced across participants (Fig. 1B). That is, twelve of the participants performed the tasks in the order: localizer, with orthosis, without orthosis; and eleven in the order: localizer, without orthosis, with orthosis.

\section{Scanning procedure}

Images were collected on a 3 T Magnetom Trio MRI scanner system (Siemens Medical Systems, Erlangen, Germany) using a 32-channel radiofrequency head coil. The structural images were obtained using a three-dimensional T1-weighted magnetisation prepared gradientecho sequence (MPRAGE) based on the ADNI protocol (www.adniinfo.org) (repetition time $(\mathrm{TR})=2500 \mathrm{~ms}$; echo time $(\mathrm{TE})=4.77 \mathrm{~ms}$; $\mathrm{TI}=1100 \mathrm{~ms}$, acquisition matrix $=256 \times 256 \times 176$, flip angle $=7^{\circ}$; $1 \times 1 \times 1 \mathrm{~mm}$ voxel size). Functional images were collected using a T2*-weighted echo planar imaging (EPI) sequence sensitive to blood oxygen level dependent (BOLD) contrast (TR $=2000 \mathrm{~ms}, \mathrm{TE}=30 \mathrm{~ms}$, image matrix $=64 \times 64$, FOV $=216 \mathrm{~mm}$, flip angle $=80^{\circ}$, voxel size $3 \times 3 \times 3 \mathrm{~mm}^{3}, 36$ axial slices).

\section{fMRI data pre-processing and main analysis}

The fMRI data were analysed using SPM8 software (Wellcome Department of Cognitive Neurology, London, UK). The first four volumes of all EPI series were excluded from the analysis to allow the magnetisation to approach a dynamic equilibrium. Data processing started with slice time correction and realignment of the EPI datasets. A mean image for all EPI volumes was created, to which individual volumes were spatially realigned by means of rigid body transformations. The structural image was co-registered with the mean image of the EPI series. Then the structural image was normalised to the Montreal Neurological Institute (MNI) template, and the normalisation parameters were applied to the EPI images to ensure an anatomically informed normalisation. A commonly applied filter of $8 \mathrm{~mm}$ FWHM (full-width at half maximum) was used. Low-frequency drifts in the time domain were removed by modelling the time series for each voxel by a set of discrete cosine functions to which a cut-off of $128 \mathrm{~s}$ was applied. The statistical analyses were performed using the general linear model (GLM). We modelled the visually presented objects separately depending on the orientation of the handle (right vs. left). These vectors were convolved with a canonical hemodynamic response function (HRF) and its temporal derivatives to form regressors in a design matrix. The parameters of the ensuing general linear model were estimated in the usual way and used to form contrasts, testing for main effects and interactions. The resulting contrast images were then entered into a series of one sample $t$-tests at the second (between-subject) level. This is the usual summary statistic approach to random effect analyses. For display purposes the resulting SPMs were thresholded at $p<0.001$ (uncorrected). A significant effect was reported when the volume of the cluster was greater than the Monte Carlo simulation determined minimum cluster size above which the probability of type I error was $<0.05$ (AlphaSim, Ward, 2000). We were primarily interested in the whole brain interaction effects of condition (with restraint, without restraint) $\times$ stimulus action side (right vs. left). The resulting maps were overlaid onto a normalised T1 weighted MNI template (colin27) and the coordinates reported correspond to the MNI coordinate system.

\section{Analysis strategy}

We used the images from the first data acquisition during the localizer task in order to determine the task-related brain regions as ROIs. For both tasks, we computed task against baseline contrasts and built ROIs of the resulting significant clusters. Within each significant functionally defined cluster (all significant voxels included) we extracted mean percent signal change over a time window of 4-6 $\mathrm{s}$ after a stimulus onset for each subject, region and condition (http://marsbar. sourceforge.net/, Brett et al., 2002). On the resulting percent signal changes we computed a repeated-measures ANOVA in search for 


\section{A) Object task}

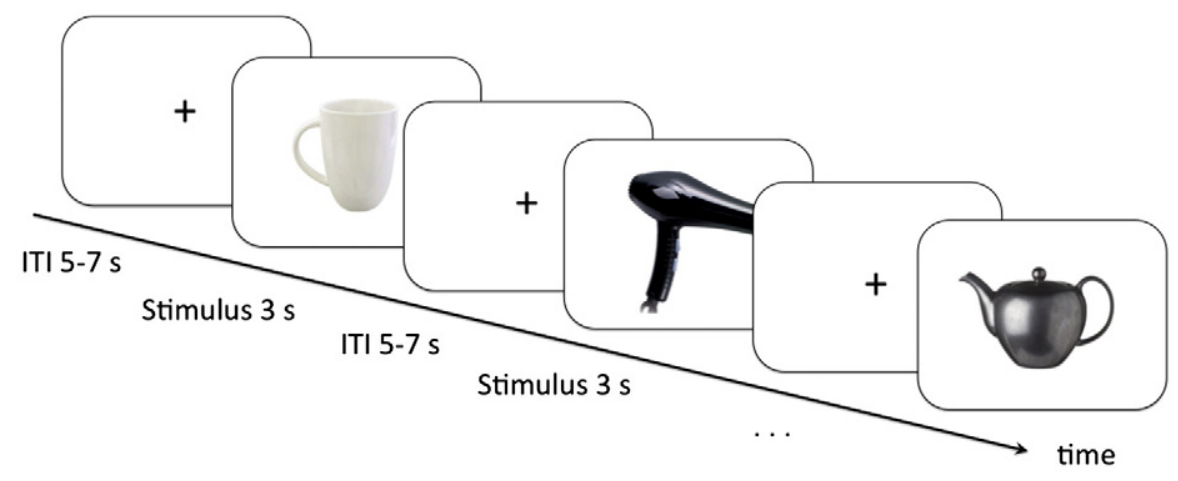

\section{B) Experimental procedure}

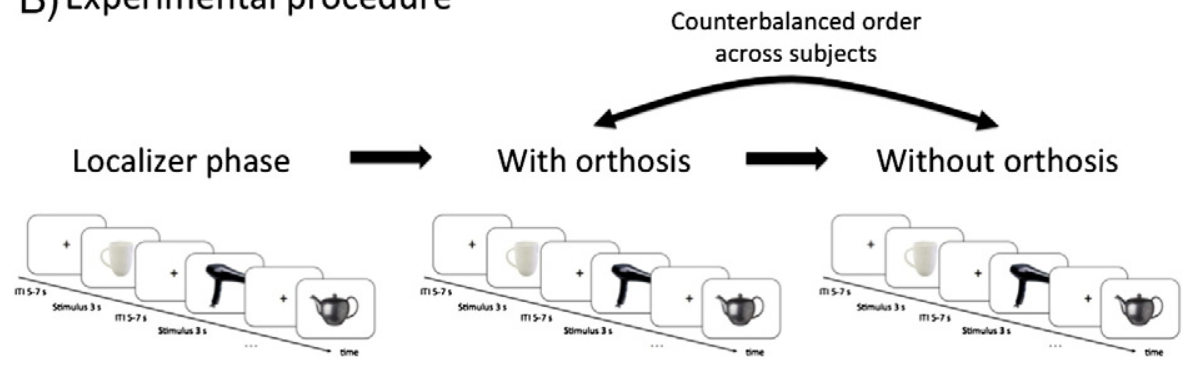

Fig. 1. (A) Schema depicting the object task; and (B) schematic drawing of the experimental procedure.

interactions of condition (with restraint, without restraint) $\times$ stimulus action side (right vs. left).

\section{Results}

We were mainly interested in brain regions showing an interaction of BOLD signal for condition (with restraint, without restraint) and stimulus action side (right vs. left). We predicted that the absence or presence of an acute restraint of the right hand should have specific effects on stimuli affording actions on the left or right side.

In a whole brain analysis on the object task, we found a significant interaction effect in the right dorsal premotor cortex $(45,8,37)$ and left cerebellum, namely lobule VI $(-33,-61,-29$, Diedrichsen, 2006). The BOLD signal changes extracted from the significant clusters are depicted in Fig. 2. Post-hoc t-tests revealed a significant difference between the right premotor BOLD activity during restraint vs. no restraint when stimuli with a handle on the left were shown $(t(22)=$ 2.22, $p=0.037$, Cohen's $d=0.32$ ) and when a handle was shown left compared to right when the right hand was restrained $(t(22)=2.98$, $p=0.007, d=0.36$ ). In the left cerebellum cluster post-hoc t-tests likewise revealed a significant difference between with and without restraint condition when a handle on the left was shown $(t(22)=2.23$, $p=0.036, d=0.52$ ) and between the left and right-side handle when the hand was not restrained $(t(22)=3.02, p=0.006, d=$ 0.35). Most importantly, the significant region in the right dorsal premotor cortex was partly overlapping with the activity observed during object task vs. baseline in the localizer. BOLD signal changes extracted from the task vs. baseline contrast in the object task likewise revealed a significant interaction in the right premotor cortex $(F(1,22)=$ $\left..544, p=0.029, \eta^{2}=0.171\right)$. The effect sizes observed are small to medium. No significant interactions (i.e., all $p$-values $>0.05$ ) were detected in the supplementary motor area $(-3,11,55)$, left ventral premotor cortex $(-48,8,31)$, bilateral parietal cortex $(26,-60,55$; $-24,-60,50)$ or bilateral visual cortex $(22,-86,-4 ;-24,-86$, $-10)(p>.46)$.

\section{Discussion}

Our results demonstrate an effect of acute immobilisation of the dominant hand on the processing of graspable objects. When the right hand was immobilised, the right dorsal premotor cortex as well as the left cerebellum were more strongly activated when the handle of a visually presented object was facing the left hand, compared to the unrestrained condition. These changes after acute immobilisation demonstrate the high speed with which neural functional reorganisation in response to changes in action requirements can take place.

The premotor cortex is generally assumed to be involved in processes related to movement preparation (Freund, 1990; Halsband et al., 1993). The dorsal premotor cortex in particular has been associated with the selection of action (Cavina-Pratesi, 2006; O'Shea et al., 2007; Toni et al., 2002) and the generation of motor plans (de Lange et al., 2005). The involvement of the dorsal premotor cortex has been shown not only for actions that are actually executed but also for the so-called "non-actions", that is, actions that are considered, but deliberately decided against (Kühn et al., 2010). Evidence for the involvement of the premotor cortex in affordances of objects is provided by an fMRI study using a repetition suppression design with repeated presentation of tools leading to reduced fMRI signal within a selective network of parietal and premotor areas (Valyear et al., 2012).

The cerebellum also plays a crucial role in motor control. It is mainly involved in the coordination, precision, and accurate timing of movement (Manto et al., 2012). The ipsilateral relationship between the lateral portions of the cerebellum and the corresponding extremities is commonly accepted, as is the topography of motor function within the cerebellum (Manni and Petrosini, 2004; Rijntjes et al., 1999). This somatotopy fits very well to the location in which the relevant interaction in the present study was observed: the cluster in lobule VI is close to the representation of the fingers of the left hand. In previous studies lobule VI has been shown to play a central role in movement preparation (Hülsmann et al., 2003). Similarly, cerebellar involvement has been observed in prehensile movements and reach-to-grasp (Grafton et al., 1996; Smith et al., 1993). 


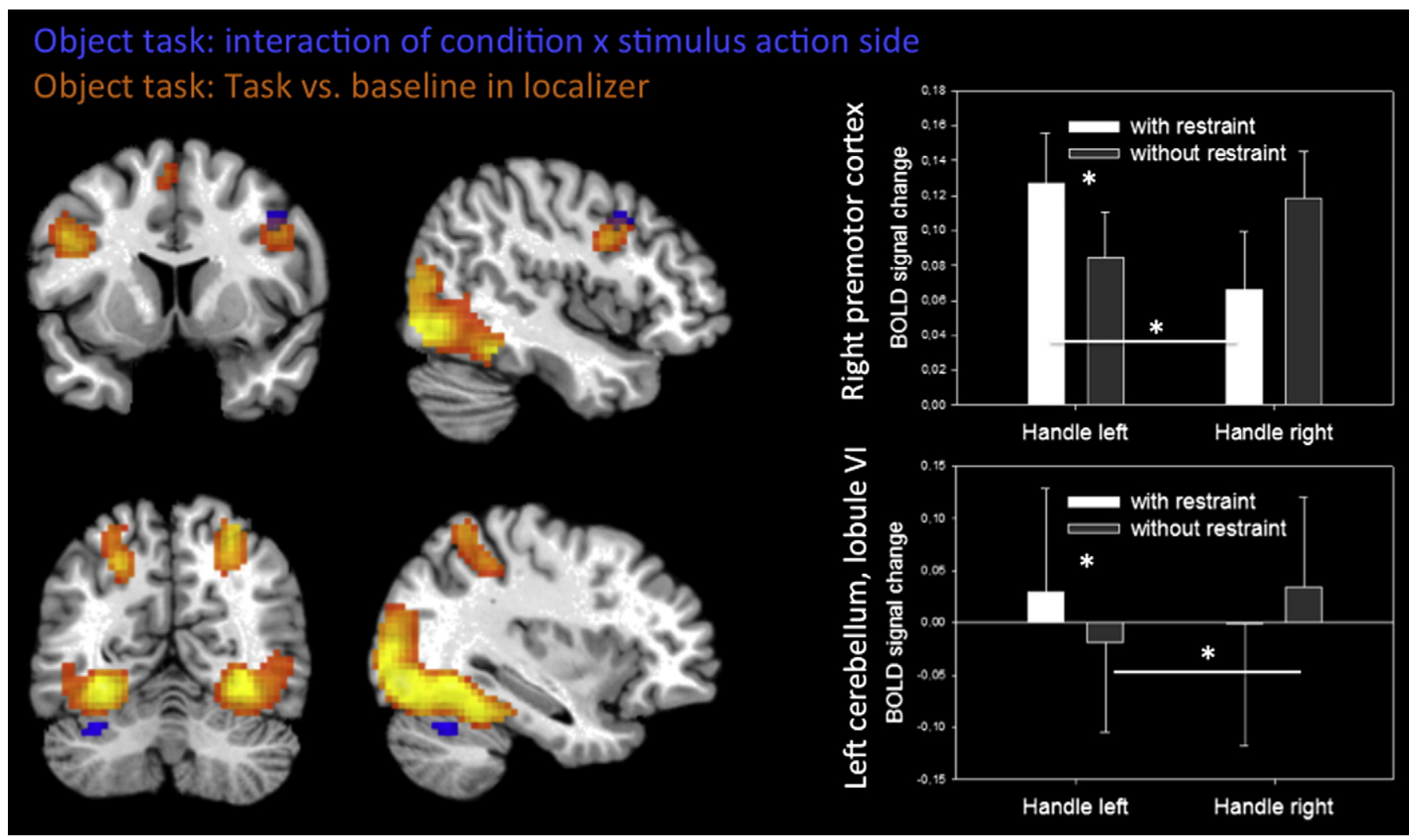

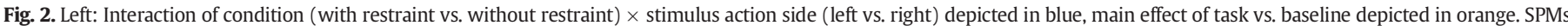

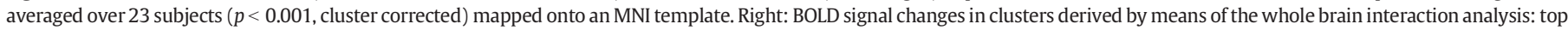
$=$ right premotor cortex, bottom $=$ left cerebellum; ${ }^{*}=$ paired $t$-test $p<0.05$.

Taken together, the location that displays the hypothesized interaction of restraint condition and side of object inviting for action supports the assumption that responses to object affordances are modulated by acute immobilisation. The motoric preparation of the left hand in the right dorsal premotor cortex and lobule VI of the left cerebellum of right handers is facilitated when an object affords an action with the left hand, when the dominant right hand is momentarily unavailable for action.

The present results show that functional reorganisation in response to altered object affordances occurs almost instantly. Previous studies that have explored the effects of short-term immobilisation restrained participants for at least $6-12 \mathrm{~h}$ in experiments focussing on behaviour (Bassolino et al., 2012; Bolzoni et al., 2012; Moisello et al., 2008) and at least for $72 \mathrm{~h}$ when focussing on neuroimaging markers (Weibull et al., 2011). That restraining movement can have instant effects has been shown in the example of tongue depression. In a previous study, we immobilised the tongue of participants by asking them to press with their tongue from the bottom against a wooden tongue spatula into the direction of the palate (Kühn and Brass, 2008); this manipulation affects the articulatory system, effectively preventing overt and subvocal speech. The behavioural evidence from this study suggests that the restraint leads to a deactivation in the articulatory system without the need of a prolonged phase of non-use.

Additional evidence for almost instantaneous effects of movement restraint in the motoric domain comes from a study in which the hands of the participants were tied behind their back (Ambrosini and Sinigaglia, 2012). The authors monitored proactive eye movements of the participants when observing an actor grasping objects and found them to be impaired when the observers' hands were restrained. In another study using a more cognitive task, wrestlers and non-athletes were asked to judge whether pairs of three-dimensional stimuli were identical or different in context of a mental rotation task, with their hands either constrained or unconstrained. Generally, wrestlers showed higher performance than controls in the rotation of geometric stimuli, but the difference disappeared when their hands were constrained suggesting that the experts' advantage in mental rotation of abstract objects was based on the readiness to act and the possibility of potential action (Moreau, 2013).

In line with these two behavioural studies, the present neuroimaging findings can be interpreted in the light of the concept of embodied cognition, implicating that cognition is situated in a particular realworld context that exerts its influence in performance. Moreover, it encompasses the notion that cognition is for action (Prinz, 1997). It acknowledges the fact that the perception of what we see is always influenced by our experience with it. This implies that seeing objects cannot be free of retrieval of the associated knowledge about how to act on these objects and spontaneously comply with its affordances. Gibson defined affordances of objects as "action possibilities" (Gibson, 1977). The present result demonstrates that the neural perceptual response to object affordances can almost instantaneously be altered when a limb can temporarily not be used. This is an interesting case of functional neuronal reorganisation, demonstrating that the association between object and related motor programs to act on this object can be flexibly modulated in face of physical restraint, even without extended experience with the behavioural consequences of the restraint. It is likely that this affordance-induced functional reorganisation leads the way to more lasting changes, including those that qualify as structural plasticity such as changes in grey matter volume or white matter connectivity.

Future research should investigate how the effects of immobilisation on object processing develop over time. For instance, one may expect that motor regions associated with left hand actions will become more dominant with prolonged immobilisation periods, so that they might respond not only to objects with an action affordance on the left side but also to objects with a handle on the right side. In constraintinduced movement therapy, the less-affected arm of patients is 
restrained in order to encourage the use of the more affected arm. This rehabilitation approach has been shown to be successful in mobilizing the non-restrained arm for spontaneous use in people with cerebral palsy or post-stroke hemiparesis (Mark et al., 2006; Taub et al., 2006). The present results suggest that this success may not merely be attributable to the actual motor training due to the forced-use of the nonrestrained arm, but also to changes in the perception of action affordances induced by the presence of the restraint as such. Studies focusing on attention have shown that graspable objects have the capacity to draw visual spatial attention to their location in the right and left hemifield, even if the objects are irrelevant for behaviour (Handy and Tipper, 2007; Handy et al., 2003). Therefore it may be interesting for future research to focus on effects of immobilisation on attention orientation.

Taken together, the present findings show that acute immobilisation of the dominant right hand leads to rapid changes in the neural processing of affordances of visually perceived objects. The right dorsal premotor cortex and the left cerebellum are more strongly activated when the handle of an object is oriented towards the left hand while the right hand is immobilised, whereas they are less activated when the handle is pointing to the right hand when the right hand can temporarily not be used. These acute immobilisation effects demonstrate that neural functional reorganisation and adaptation can take place at high speed.

\section{Acknowledgments}

We are grateful for the assistance of the MRI team at the MPI Berlin consisting of Sonali Beckmann, Nils Bodammer, Thomas Feg, Sebastian Schröder, Nadine Taube.

\section{References}

Ambrosini, E., Sinigaglia, C., 2012. Tie my hands, tie my eyes. J. Exp. Psychol. Hum. Percept Perform. 38, 263-266.

Avanzino, L., Bassolino, M., Pozzo, T., Bove, M., 2011. Use-dependent hemispheric balance. J. Neurosci. 31, 3423-3428.

Bassolino, M., Bove, M., Jacono, M., Fadiga, L., Pozzo, T., 2012. Functional effect of shortterm immobilization: kinematic changes and recovery on reaching-to-grasp. Neuroscience 215, 127-134.

Bolzoni, F., Bruttini, C., Esposti, R., Cavallari, P., 2012. Hand immobilization affects arm and shoulder postural control. Exp. Brain Res. 220, 63-70.

Brett, M., Anton, J.-C., Valabreuge, R., Poline, J.B., 2002. Region of interest analysis using an SPM toolbox. Presented at the 8th International Conference on Functional Mapping of the Human Brain.

Castiello, U., 1999. Mechanisms of selection for the control of hand action. Trends Cogn. Sci. 3, 264-271.

Cavina-Pratesi, C., 2006. Dissociating arbitrary stimulus-response mapping from movement planning during preparatory period: evidence from event-related functional magnetic resonance imaging. J. Neurosci. 26, 2704-2713.

Clark, B.C., Taylor, J.L., Hoffman, R.L., Dearth, D.J., Thomas, J.S., 2010. Cast immobilization increases long-interval intracortical inhibition. Muscle Nerve 42, 363-372.

Coert, J.H., Stenekes, M.W., Paans, A.M.J., Nicolai, J.-P.A., De Jong, B.M., 2009. Clinical implications of cerebral reorganisation after primary digital flexor tendon repair. J. Hand Surg. 34, 444-448.

Craighero, L., Fadiga, L., Umiltà, C.A., Rizzolatti, G., 1996. Evidence for visuomotor priming effect. Neuroreport 8, 347

de Lange, F.P., Hagoort, P., Toni, I., 2005. Neural topography and content of movement representations. J. Cogn. Neurosci. 17, 97-112.

Diedrichsen, J., 2006. A spatially unbiased atlas template of the human cerebellum. NeuroImage 33, 127-138.

Facchini, S., Romani, M., Tinazzi, M., Aglioti, S.M., 2002. Time-related changes of excitability of the human motor system contingent upon immobilisation of the ring and little fingers. Clin. Neurophysiol. 113, 367-375.

Freund, H.J., 1990. Premotor area and preparation of movement. Rev. Neurol. 146, 543-547.
Gentilucci, M., 2002. Object motor representation and reaching-grasping control. Neuropsychologia 40, 1139-1153.

Gibson, J.J., 1977. The theory of affordances. In: Shaw, R., Bransford, J. (Eds.), Perceiving, Acting, and Knowing. Toward an Ecological Psychology. Lawrence Erlbaum Associates, Hillsdale: NJ.

Grafton, S.T., Arbib, M.A., Fadiga, L., Rizzolatti, G., 1996. Localization of grasp representations in humans by positron emission tomography. Exp. Brain Res. 112, 103-111.

Granert, O., Peller, M., Gaser, C., Groppa, S., Hallett, M., Knutzen, A., Deuschl, G., Zeuner, K.E., Siebner, H.R., 2011. Manual activity shapes structure and function in contralateral human motor hand area. Neurolmage 54, 32-41.

Grèzes, J., Tucker, M., Armony, J., Ellis, R., Passingham, R.E., 2003. Objects automatically potentiate action: an fMRI study of implicit processing. Eur. J. Neurosci. 17, 2735-2740.

Halsband, U., Ito, N., Tanji, J., Freund, H.J., 1993. The role of premotor cortex and the supplementary motor area in the temporal control of movement in man. Brain 116, 243-266.

Handy, T.C., Tipper, C.M., 2007. Attentional orienting to graspable objects: what triggers the response? Neuroreport 18, 941-944.

Handy, T.C., Grafton, S.T., Shroff, N.M., Ketay, S., Gazzaniga, M.S., 2003. Graspable objects grab attention when the potential for action is recognized. Nat. Neurosci. 6, 421-427.

Hülsmann, E., Erb, M., Grodd, W., 2003. From will to action: sequential cerebellar contributions to voluntary movement. Neurolmage 20,1485-1492.

Kühn, S., Brass, M., 2008. Testing the connection of the mirror system and speech. Neuropsychologia 46, 1513-1521.

Kühn, S., Bodammer, N.C., Brass, M., 2010. Dissociating mental states related to doing nothing by means of fMRI pattern classification. NeuroImage 53, 1294-1300.

Langer, N., Hänggi, J., Müller, N.A., Simmen, H.P., Jäncke, L., 2012. Effects of limb immobilization on brain plasticity. Neurology $78,182-188$.

Lecrubier, Y, Sheehan, D.V., Weiller, E, Amorim, P., Bonora, I., Harnett Sheehan, K, Janavs, J., Dunbar, G.C., 1997. The Mini International Neuropsychiatric Interview (MINI). A short diagnostic structured interview: reliability and validity according to the CIDI. Eur. Psychiatry 12, 224-231.

Lissek, S., Wilimzig, C., Stude, P., Pleger, B., Kalisch, T., Maier, C., Peters, S.A., Nicolas, V. Tegenthoff, M., Dinse, H.R., 2009. Immobilization impairs tactile perception and shrinks somatosensory cortical maps. Curr. Biol. 19, 837-842.

Manni, E., Petrosini, L., 2004. A century of cerebellar somatotopy: a debated representation. Nat. Rev. Neurosci. 5, 241-249.

Manto, M., Bower, J.M., Conforto, A.B., Delgado-García, J.M., da Guarda, S.N.F., Gerwig, M., Habas, C., Hagura, N., Ivry, R.B., Mariën, P., Molinari, M., Naito, E., Nowak, D.A., Oulad Ben Taib, N., Pelisson, D., Tesche, C.D., Tilikete, C., Timmann, D., 2012. Consensus paper: roles of the cerebellum in motor control-the diversity of ideas on cerebellar involvement in movement. Cerebellum 11, 457-487.

Mark, V.W., Taub, E., Morris, D.M., 2006. Neuroplasticity and constraint-induced movement therapy. Eura Medicophys. 42 (3), 269-284.

Moisello, C., Bove, M., Huber, R., Abbruzzese, G., Battaglia, F., Tononi, G., Ghilardi, M.F., 2008. Short-term limb immobilization affects motor performance. J. Mot. Behav. 40, $165-176$.

Moreau, D., 2013. Constraining movement alters the recruitment of motor processes in mental rotation. Exp. Brain Res. 224, 447-454.

Ngomo, S., Leonard, G., Mercier, C., 2012. Influence of the amount of use on hand motor cortex representation: effects of immobilization and motor training. Neuroscience 220, 208-214.

Oldfield, R.C., 1971. The assessment and analysis of handedness: the Edinburgh inventory. Neuropsychologia 9, 97-113.

O'Shea, J., Johansen-Berg, H., Trief, D., Göbel, S., 2007. Functionally specific reorganization in human premotor cortex. Neuron 54, 479-490.

Pascual-Leone, A., Amedi, A., Fregni, F., Merabet, L.B., 2005. The plastic human brain cortex. Annu. Rev. Neurosci. 28, 377-401.

Prinz, W., 1997. Perception and action planning. Eur. J. Cogn. Psychol. 9, 129-154.

Rijntjes, M., Dettmers, C., Büchel, C., Kiebel, S., Frackowiak, R.S.J., Weiller, C., 1999. A blueprint for movement: functional and anatomical representations in the human motor system. J. Neurosci. 19, 8043-8048.

Smith, A.M., Dugas, C., Fortier, P., Kalaska, J., 1993. Comparing cerebellar and motor cortical activity in reaching and grasping. Can. J. Neurol. Sci. 20, S53-S61.

Taub, E., Uswatte, G., Mark, V.W., Morris, D.M., 2006. The learned nonuse phenomenon: implications for rehabilitation. Eura Medicophys. 42, 241-255.

Toni, I., Shah, N.J., Fink, G.R., Thoenissen, D., Passingham, R.E., Zilles, K., 2002. Multiple movement representations in the human brain: an event-related fMRI study. J. Cogn. Neurosci. 14, 769-784.

Valyear, K.F., Gallivan, J.P., McLean, D.A., Culham, J.C., 2012. fMRI repetition suppression for familiar but not arbitrary actions with tools. J. Neurosci. 32, 4247-4259.

Ward, B.D., 2000. Simultaneous inference for fMRI data. AFNI AlphaSim Documentation. Medical College of Wisconsin.

Weibull, A., Flondell, M., Rosén, B., Björkman, A., 2011. Cerebral and clinical effects of short-term hand immobilisation. Eur. J. Neurosci. 33, 699-704. 